\title{
Coriocarcinoma con metástasis a Pulmón
}

\section{Choriocarcinoma metastases to Lung}

\author{
Fabio Rojas ${ }^{1 *}$, Susana Martínez², Yordiana Medina ${ }^{2}$
}

\begin{abstract}
Resumen
El coriocarcinoma es un tumor maligno que se caracteriza por hiperplasia trofoblástica con anaplasia, ausencia de vellosidades, hemorragia y necrosis. Se encuentra una paciente con antecedentes de mola hidatidiforme que, después de tres años, se le diagnosticó coriocarcinoma con metástasis a pulmón. La paciente consultó por amenorrea y dolor abdominal, se le iniciaron estudios encontrando masa uterina. El análisis patológico reportó coriocarcinoma. Posterior al diagnóstico la paciente inició tratamiento quimioterapéutico con evolución adecuada y curación completa. El coriocarcinoma carece vellosidades coriales, sincitio y citotrofoblasto anormal, presenta hemorragia y necrosis que invade el miometrio y los vasos sanguíneos. Hace metástasis principalmente a pulmón, cerebro e hígado, se registra mortalidad menor al $15 \%$ en pacientes con diagnóstico temprano y tratamiento oportuno; se ha demostrado que es un fumor ampliamente quimiosensible $y$, a pesar de la presencia de metástasis, existe una alta tasa de curación.

Palabras clave: Enfermedad trofoblástica gestacional, mola hidatidiforme, coriocarcinoma, quimioterapia.

Abstract

It is a patient with a history of hydatidiform mole that after three years was diagnosed with choriocarcinoma metastasis lung. The patient consulted by amenorrhea and abdominal pain. The medical staff began studies finding uterine mass which took pathological study that reported an following that, the patient started chemotherapeutic treatment with a suitable evolution and complete healing. The choriocarcinoma in a rare entity characterized by the absence of chorionic villi, syncytium and abnormal cytotrophoblast, with hemorrhage and necrosis that invades the myometrium and blood vessels. Its main serological marker is the smoked persistent chorionic gonadotropin. This can metastasize mainly in lung, brain, and liver. Lower mortality is recorded at $15 \%$ in patients who underwent early diagnosis and timely treatment. It has been shown to be a highly chemosensitive tumor, and therefore, it has a high response despite the presence of metastasis, but it has a high rate of cure.

Keywords: Gestational trophoblastic disease, hydatidiform mole, choriocarcinoma, chemotherapy.
\end{abstract}

\section{Introducción}

El coriocarcinoma es un tumor maligno que se caracteriza por hiperplasia trofoblástica con anaplasia, ausencia de vellosidades, hemorragia y necrosis. Se considera una neoplasia curable pero si no recibe tratamiento oportuno es mortal. La neoplasia trofoblástica gestacional se considera el tumor ginecológico más curable debido a dos características fundamentales: es altamente sensible a la quimioterapia y es productor de la fracción beta de la Gonadotrofina Coriónica Humana (beta-hGC), la cual es un efectivo marcador que permite diagnosticar la enfermedad y monitorear la terapia ${ }^{[1]}$. Clínicamente, las manifestaciones más importantes son el sangrado transvaginal asociado a un crecimiento uterino mayor a la amenorrea ${ }^{[2]}$. La piedra angular del tratamiento es la quimioterapia de uno o varios agentes. El objetivo del presente documento es presentar un caso de coriocarcinoma en una mujer joven multigestante con historia obstétrica adversa y metástasis a pulmón.

\section{Presentación del Caso}

Paciente femenina de 31 años de edad G5A5VO MOLA 1 (MOLA hace aproximadamente 3 años) con cuadro clínico de 4 meses de evolución de nauseas, mareos, asociado a dolor

1. Ginecólogo y Obstetra. Coordinador de Postgrado de Ginecología y Obstetricia, Universidad Surcolombiana

2. Residente de Ginecología y Obstetricia, Universidad Surcolombiana.

* Correspondencia: Fabio Rojas. Correo electrónico: farojas@hotmail.com Recepción: 15/02/2016. Revisado: 04/08/2016. Aceptación: 14/11/2016 
en región pélvica de forma intermitente; motivo por el cual consultó a institución de primer nivel, donde realizó prueba cualitativa de embarazo positiva, con posterior ecografía transvaginal que evidenciaba útero y anexos normales. Dada la persistencia del cuadro clínico se realizó nueva ecografía, en la cual se evidencia una imagen sugestiva de mioma intramural (figura 1), sin signos ecográficos de embarazo ni extra ni intrauterino. Como estudio complementario la hormona gonadotropina coriónica humana (BhCG) que se encontraba positiva y en meseta (figura 2).

Después de varios estudios de imágenes, y teniendo en cuenta que no se llegó a un diagnóstico definitivo, se realizó laparoscopia diagnóstica, en la que se evidenció útero con masa de $3 \times 4 \mathrm{~cm}$ en región fundica (figura 3), con centro hemorrágico visualizado a través de la serosa, adherida a colon sigmoides. En ese momento se consideró imagen compatible clínicamente con embarazo ectópico cornual, con posterior laparotomía exploratoria y resección de masa uterina (se realizó respectivo estudio histopatológico) (figura 4), reportando neoplasia maligna epitelioide de alto grado, pobremente diferenciado. Posteriormente se realizó estudio de inmunohistoquímica que reportó coriocarcinoma con índice de proliferación celular KI-90\%. Dados los hallazgos histopatológicos, se realizaron estudios de extensión con reporte de TAC de abdomen simple y contrastada, donde se evidenciaron imágenes sugestivas de metástasis en bases pulmonares; se indicó tratamiento quimioterapéutico con etopósido días 1 y 2, $189 \mathrm{mg}$, metrotrexate $189 \mathrm{mg}$ día 2, actinomicina D $0,5 \mathrm{mg}$ IV día 2 y 7 , ciclofosfamida $1134 \mathrm{mg}$ IV día 1 , vincristina $2 \mathrm{mg}$ IV día 1, 4 ciclos; los controles clínicos y paraclínicos evidenciaron curación con negativización de la BhCG.

\section{Conceptos}

En el año 2000, la Federación Internacional de Ginecología y Obstetricia (FIGO), introdujo el término neoplasia trofoblástica gestacional, condición en la cual los niveles de hCG fallan en caer en la ausencia de un embarazo normal ${ }^{[3]}$. Los criterios diagnósticos al menos uno de estos: a) hCG persistentemente elevada o en meseta por al menos 4 valores (día 1, 7, 14 y 21); b) aumento progresivo o mayor del $10 \%$ de hCG por 2 semanas o más; c) persistencia de hCG después de 6 meses de la evacuación; d) diagnóstico histopatológico de coriocarcinoma; y e) metástasis pulmonares diagnosticadas por rayos $\mathrm{X}$ de tórax.

Incluye patologías como coriocarcinoma, mola invasora, tumor del sitio de inserción placentario y tumor trofoblástico epitelial ${ }^{[4]}$. El coriocarcinoma en una entidad rara, se caracteriza por la ausencia de vellosidades coriales, sincitio y citotrofoblasto anormal, con hemorragia y necrosis que invade el miometrio y los vasos sanguíneos ${ }^{[2]}$.

Epidemiología: Se estima que el coriocarcinoma se presenta 1 de cada 50000 embarazos, y en el sureste asiático,

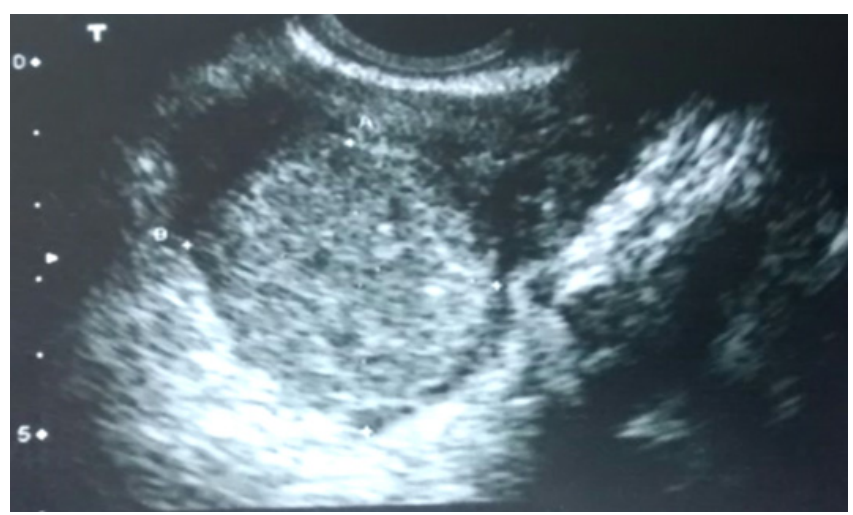

Figura 1. Imagen ultrasonográfica de la masa

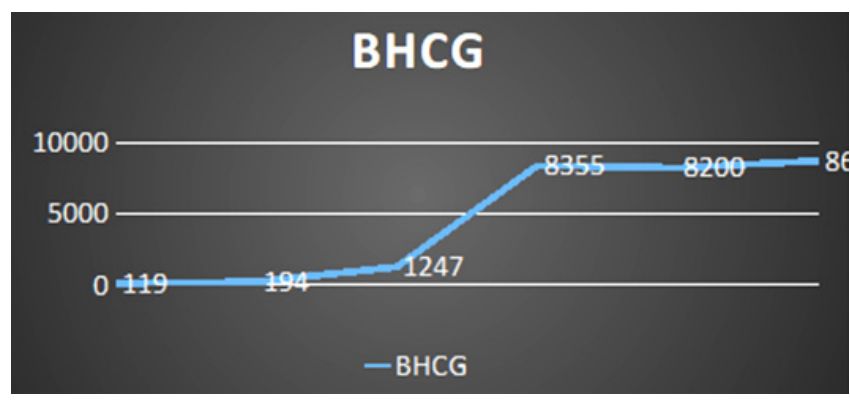

Figura 2. Seguimiento de BhCG $\mathrm{mlU} / \mathrm{mL}$

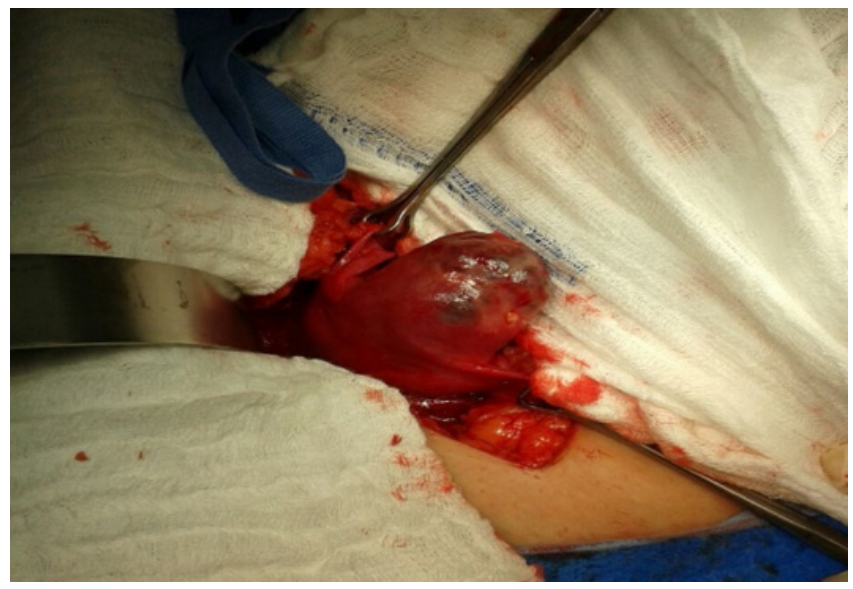

Figura 3. Visualización macroscópica de la masa

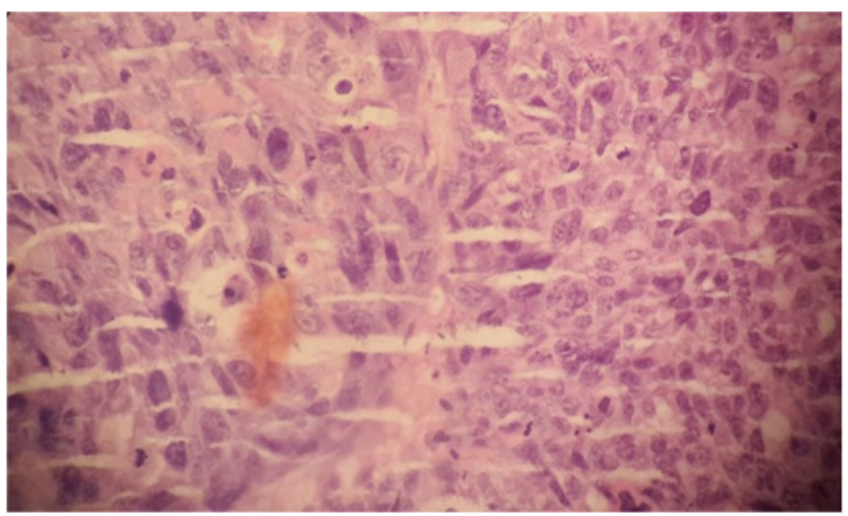

Figura 4. Visualización microscópica 
9 de cada 50000 embarazos ${ }^{[2,4,5]}$. Los factores de riesgo asociados son aborto, embarazo ectópico, embarazo a término y mola hidatiforme. La edad materna, la etnia, antecedente de embarazo molar y niveles de hCG elevados post evacuación están asociados con muy alto riesgo de neoplasia trofoblástica. El uso de anticonceptivos orales no se ha llegado a consenso con respecto a aumento del riesgo ${ }^{[6,7]}$.

- Metástasis y neoplasia trofoblastica gestacional: Los sitios de metastásis más comunes son pulmón (80\%), vagina (30\%), cerebro (10\%), y el hígado (10\%). La biopsia de las metástasis no es necesaria ni recomendada debido al riesgo de hemorragia ${ }^{[8]}$.

- Tratamiento: Se basa en la clasificación del grupo de riesgo de la paciente definido por el estadio y por el score en la escala de riesgo (ver tabla 1 y 2$)^{[1,9]}$.

- Indicaciones de quimioterapia ${ }^{[1-14]}$ : Metástasis cerebrales, hepáticas, gastrointestinales o pulmonares $>2$ $\mathrm{cm}$. Evidencia histológica de coriocarcinoma, sangrado profuso a nivel vaginal, gastrointestinal o intraperitoneal. Metástasis pulmonar, vaginal o vulvar en el cual los niveles de hCG no descienden, elevación de hCG en 2 muestras de suero consecutivas, niveles séricos de hCG >20000 IU/1 después de 4 semanas post evacuación, niveles de hCG en meseta en 3 muestras de suero consecutivas luego de la evacuación y elevación de hCG 6 meses después de la evacuación aun si esta ha bajado.

- $\quad$ Pacientes de bajo riesgo: Se ha utilizado ampliamente el metrotexate en varios regímenes. La tasas de respuesta completa es $72 \%$ y, menos del $5 \%$ de los pacientes desarrollan toxicidad grave. Actinomicina D es otra droga usada. El $80 \%$ de los pacientes resistentes al metrotexate tiene respuesta completa a este esquema de tratamiento ${ }^{[1]}$.

- Pacientes de alto riesgo: Varias combinaciones de medicamentos se han descrito.

EMA-CO: se aplica en día 1 etopósido, actinomicina D, metrotexate. Día 2: etopósido, actinomicina $\mathrm{D}$ y rescate con ácido folínico. Día 8: vincristina y ciclofosfamida. La tasa de éxito de este esquema es cercano al $85 \%$ y la tasa total de supervivencia es cercana al 90\% ${ }^{[1,2,4,5]}$. Otros esquemas de tratamiento: MEA (metrotexate, etopósido y actinomicina D), MAC (metrotexate, actinomicina D y ciclofosfamida o clorambucil), CHAMOMA (ciclofosfamida, hidroxiurea actinomicina $\mathrm{D}$, metrotexate con acido folínico, vincristina $\mathrm{y}$ doxorubicina), CHAMOC (ciclofosfamida, hidroxiurea, actinomicina D, Metrotexate con acido folinico y vincristina) y EP - EMA (etopósido, cisplatino, metrotexate y actinomicina $\mathrm{D}$ ). La tasa de respuesta es de $60-80 \%$, cuando se usan como primera línea ${ }^{[1,3-5]}$.

- Persistencia o recurrencia de neoplasia trofoblástica de alto riesgo: La tasa de recurrencia es del 3-10\%. Cerca del $20 \%$ de estos pacientes no responden al tratamiento y mueren. La tasa de supervivencia total en pacientes con recurrencia es $85-90 \%{ }^{[4,11]}$.

- Rol de la cirugía: indicada para remover enfermedad persistente o recurrente en el útero o sitios de metástasis, control de hemorragias tumorales, disminución del

Tabla 1. Estatificación anatómica para la neoplasia trofoblastica gestacional - FIGO

\begin{tabular}{ll}
\hline Estadio & Definición \\
\hline I & Enfermedad confinada al útero. \\
II & Neoplasia extendida a otros sitios fuera del útero, pero limitada a estructuras genitales (anexos, vagina, \\
& ligamento ancho). \\
III & Neoplasia trofoblastica gestacional extendida a pulmones, con o sin compromiso genital. \\
IV & Metástasis a otros sitios \\
\hline
\end{tabular}

Tabla 2. Sistema pronóstico de la OMS modificado por la FIGO

\begin{tabular}{lcccc}
\hline Puntuación & $\mathbf{0}$ & $\mathbf{1}$ & $\mathbf{2}$ & $\mathbf{4}$ \\
\hline Edad & $<40$ & $=40$ & - & - \\
Antecedente de embarazo & Mola & Aborto & Termino & - \\
Periodo intergenesico & $<4$ & $4-<7$ & $7-<13$ & - \\
Niveles de hCG pre tratamiento & $<103$ & $103-<104$ & $104-105$ & $=105$ \\
Tamaño del tumor & - & $3-<5 \mathrm{~cm}$ & $=5 \mathrm{~cm}$ & - \\
Sitio de metástasis & Pulmón & Bazo, riñón & Gastrointestinal & Hígado, cerebro \\
Número de metástasis & - & $1-4$ & $5-8$ & $>8$ \\
Falla de quimioterapia previa & - & - & Solo una droga & $=2$ drogas \\
\hline
\end{tabular}


tamaño tumoral, tratar infecciones y alivio de síntomas como obstrucción intestinal o urinaria $[4,13]$.

- Rol de la radioterapia: Es principalmente usada para tratar metástasis cerebrales y prevenir sangrados súbitos, cuando se realiza irradiación cerebral y quimioterapia concomitante las tasas de supervivencia son $40-90 \%$.

- Seguimiento: Las recurrencias ocurren principalmente en el primer año. No hay un esquema de seguimiento específico, sin embargo el más sugerido es determinación de HCG sérica y urinaria 2 veces por semana durante el tratamiento. Finalizado el tratamiento se hacen determinaciones semanales por 6 semanas. A las 6 semanas pos-tratamiento se debe realizar ecografía-doppler de la pelvis, tomografía o resonancia si hay alguna manifestación anómala.

- Anticoncepción y embarazos posteriores: Se recomienda anticoncepción al menos 12 meses después de haber terminado el tratamiento ${ }^{[4,14]}$. Los pacientes con antecedentes de neoplasia trofoblástica gestacional pueden tener resultados reproductivos normales ${ }^{[8,10,14,15]}$.

\section{Discusión}

El coriocarcinoma es la patología del trofoblasto altamente agresiva, por su gran capacidad de angioinvasión, lo que facilita su diseminación y propicia el desarrollo de metástasis pulmonares, cerebrales, hepáticas y a otros órganos ${ }^{[1]}$. Puede ser fatal, pues aunque se diagnostique oportunamente y reciba tratamiento con quimioterapia en forma adecuada, tiene una mortalidad de $10 \%$ a $15 \%{ }^{[4]}$. En nuestro medio, la mortalidad es mayor con base en el diagnóstico y su tratamiento, habitualmente no se realiza en forma temprana o es incompleto ${ }^{[8]}$. La sintomatología aparece tardíamente derivada de metástasis a diferentes órganos. Es importante recalcar la importancia que tiene la cuantificación sérica de la fracción beta-hCG, ya que no sólo es la base del diagnóstico, sino además establece el pronóstico y respuesta al tratamiento ${ }^{[10]}$. Desde el punto de vista etiológico, se asocia con la edad, historia de pérdidas gestacionales, el uso de anticonceptivos orales y factores ambientales o socioeconómicos, no bien establecidos ${ }^{[2]}$. La tasa de supervivencia a 5 años en pacientes con neoplasia trofoblastica gestacional es $>95 \%$ en pacientes con tratamiento adecuado y oportuno ${ }^{[15]}$.

\section{Conclusiones}

El caso presentado es claro ejemplo de las complicaciones que conlleva un embarazo. La paciente con historia obstétrica adversa, dada por las múltiples perdidas gestacionales, el antecedente de mola hidatidiforme y la progresión a coriocarcionoma con compromiso a órganos lejanos como el pulmón, que pueden causar importante morbi-mortalidad en mujeres jóvenes. Se recomienda que las pacientes con historia obstétrica adversa cuenten con seguimiento y acompañamiento, lo que asegura el éxito del tratamiento y la curación de dichas patologías.

\section{Referencias}

1. Lurain, J.R., Gestational trophoblastic disease II: classification and management of gestational trophoblastic neoplasia. Am J Obstet Gynecol, 2011. 204(1): p. 11-8 DOI: 10.1016/i.ajog.2010.06.072

2. Lurain, J.R., Gestational trophoblastic disease I: epidemiology, pathology, clinical presentation and diagnosis of gestational trophoblastic disease, and management of hydatidiform mole. Am J Obstet Gynecol, 2010. 203(6): p. 531-9 DOI: 10.1016/i.ajog.2010. 06.073

3. Soper, J.T., et al., Diagnosis and treatment of gestational trophoblastic disease: ACOG Practice Bulletin No. 53. Gynecol Oncol, 2004. 93(3): p. 575-85 DOI: 10.1016/ i.ygyno.2004.05.013

4. Tse, K.Y., et al., An update on gestational trophoblastic disease. Obstetrics, Gynaecology \& Reproductive Medicine, 2012. 22(1): p. 7-15 DOI: https://doi.org/10. 1016/i.ogrm.2011.10.004

5. Deng, L., et al., Combination chemotherapy for highrisk gestational trophoblastic tumour. Cochrane Database Syst Rev, 2009(2): p. CD005196 DOI: https://doi.org/ 10.1002/14651858.CD005196.pub3

6. Sebire, N.J. and M.J. Seckl, Gestational trophoblastic disease: current management of hydatidiform mole. BMJ, 2008. 337: p. a1193 DOI: 10.1136/bmi.a1193

7. Costa, H.L. and P. Doyle, Influence of oral contraceptives in the development of post-molar trophoblastic neoplasia-a systematic review. Gynecol Oncol, 2006. 100(3): p. 579-85 DOI: 10.1016/i.ygyno.2005.09.031

8. Berkowitz, R.S. and D.P. Goldstein, Current management of gestational trophoblastic diseases. Gynecol Oncol, 2009. 112(3): p. 654-62 DOI: 10.1016/i.ygyno.2008. 09.005

9. Lurain, J.R., et al., Prognostic factors in gestational trophoblastic tumors: a proposed new scoring system based on multivariate analysis. Am J Obstet Gynecol, 1991. 164(2): p. 611-6

10. Matsui, H., et al., Early pregnancy outcomes after chemotherapy for gestational trophoblastic tumor. J Reprod Med, 2004. 49(7): p. 531-4

11. Newlands, E.S., The management of recurrent and drugresistant gestational trophoblastic neoplasia (GTN). Best Pract Res Clin Obstet Gynaecol, 2003. 17(6): p. 905-23

12. Petersen, R.W., et al., The impact of molar pregnancy on psychological symptomatology, sexual function, and quality of life. Gynecol Oncol, 2005. 97(2): p. 535-42 DOI: 10.1016/i.ygyno.2005.01.015

13. Soper, J.T., Role of surgery and radiation therapy in the management of gestational trophoblastic disease. Best Pract Res Clin Obstet Gynaecol, 2003. 17(6): p. 943-57

14. Tse, K.Y. and H.Y. Ngan, Gestational trophoblastic disease. Best Pract Res Clin Obstet Gynaecol, 2012. 26(3): p. 357-70 DOI: 10.1016/i.bpobgyn.2011.11.009

15. Woolas, R.P., et al., Influence of chemotherapy for gestational trophoblastic disease on subsequent pregnancy outcome. Br J Obstet Gynaecol, 1998. 105(9): p. 1032-5. 